\title{
Transnasal endoscopy - Evaluation and treatment method for patients with sinus lift and dental implants indications
}

\author{
Bucharest, Romania \\ 2"Carol Davila" University of Medicine and Pharmacy, Bucharest, Romania \\ ${ }^{3}$ ENT\&HNS Department, "Sfanta Maria” Hospital, Bucharest, Romania
}

Anca Delia Vereanu ${ }^{1,2}$, Manuela Andra Savu ${ }^{3}$, Elena Patrascu ${ }^{2,3}$, Codrut Sarafoleanu ${ }^{2,3}$

1"Oral Implantology Department, "Prof. Dr. D. Theodorescu" Oro-Maxillofacial Surgery Hospital,

\section{ABSTRACT}

BACKGORUND. Sinus floor elevation, also known as sinus lift, is the surgical procedure meant to elevate the maxillary floor in order to increase the height of the alveolar bone. This will allow the oral surgeon specialist to insert the desired implants, the ground of dental reconstruction. As the procedure involves the maxillary sinus and its Schneiderian membrane the main concern is to have a healthy maxillary sinus prior to implantation and to the maxillary augmentation.

OBJECTIVE. The aim of our study was to identify the patients with abnormal CT scan and refer them to an ENT evaluation, in order to identify and treat any sinusal pathology that can lead to a poor outcome of the sinus-lift intervention.

MATERIAL AND METHODS. Our prospective study included 15 patients selected from a group of 79 adults, candidates for dental implantation with sinus augmentation. These patients had abnormal sinus CT scan in terms of mucosal hypertrophy, cysts or fungal material. Transnasal diameatic sinusoscopy was performed for all the patients, assessing the aspect of the sinus cavity and the mucosa. The following abnormal aspects were found in our group: 5 patients with cysts, 2 patients with fungus ball and 4 with mucosal hypertrophy. 4 patients had normal maxillary mucosa despite the CT scan appearance.

RESULTS. All the patients with abnormal aspects at sinusoscopy (11 patients) were operated on before sinus lift procedure antrostomy, cyst removal, fungus ball removal. 4 patients showed normal aspect of the sinus mucosa despite the CT scan donations, meaning a $26.66 \%$ error rate in appreciating the real changes of the maxillary mucosa on the CT scan.

CONCLUSION. Endoscopic assessment of the maxillary sinus is mandatory in patients with changes of the CT scan. This allows a proper examination of the sinus mucosa and can predict the outcomes of the sinus lift procedures. It is mandatory to cure the inflammation or infection of the maxillary sinus prior to implant procedure in order to be able to increase the success rate, as it can lead to failures of the implant procedure.

KEYWORDS: sinusoscopy, endoscopy, sinusal mucosa, chronic sinusitis, sinus augmentation

\section{INTRODUCTION}

Caries, and its sequelae, remain the main reason for all dental loss ${ }^{1}$. Monitoring the occurrence of an oral "end state" such as edentulism is important because it is an indicator of both population health and of a country's oral health care system ${ }^{2}$. Even if the prevalence of edentulism has declined over the last decade, it remains a major disease spread worldwide, especially among older adults ${ }^{3,4}$.

After tooth loss, the alveolar process of the maxilla resorbs in two directions: vertically and horizontally, becoming progressively smaller ${ }^{5-8}$. The loss of teeth in the posterior maxilla induces an enhancement of the maxillary sinus called (hyper)pneumatization of the sinus. The tendency towards expansion is significantly higher after molar extraction ${ }^{9,10}$ (Figure 1).

In the past years, implant dentistry developed continuously, joining the modern age of medicine and allowing doctors to reestablish the patient's oral functionality by creating a closest to normal denture. Dental implants are now widespread well-documented treatment for replacing missing teeth ${ }^{11}$.

A proper bone quality and width is necessary for a good implant result. In the preoperative evaluation the oro-maxillofacial (OMF) surgeon will decide if it is suitable to insert the implants in the existing alveolar bone, 


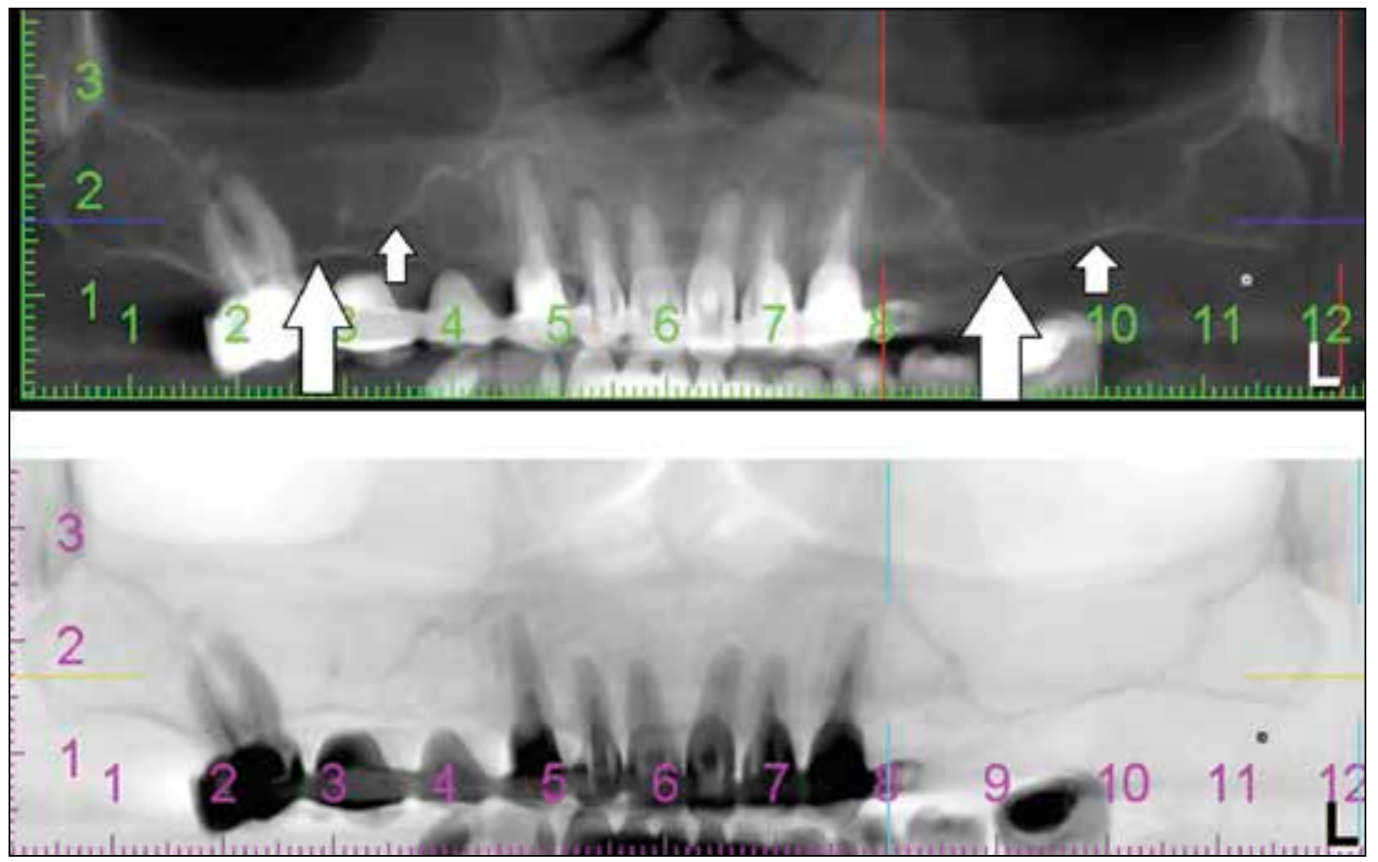

Figure 1 CT-scan - enlarged maxillary sinuses: lowered maxillary floor, generating large, hyperpneumatises sinuses (white arrows)

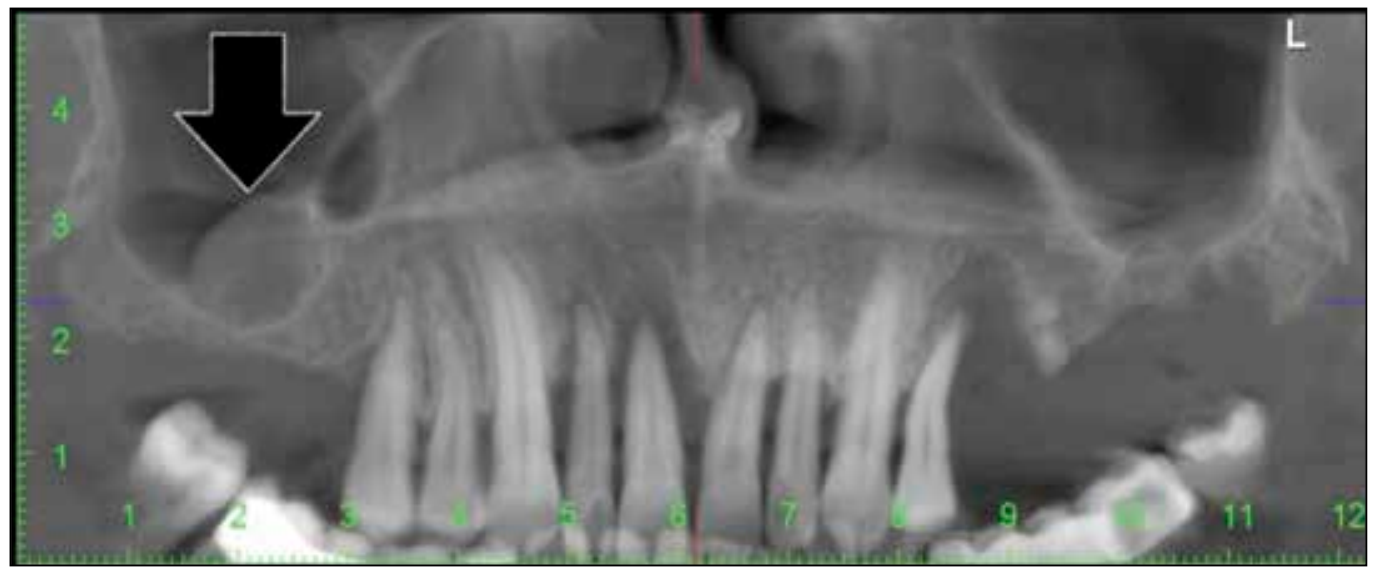

Figure 2 OPG - right maxillary cyst located in the alveolar recess - prior of implant placement this entity must be removed and the alveolar crest increased with bone addition

or if grafting is imperative. In the same phase, after performing an orthopantomography (OPG) (Figure 2) or/and computed tomography (CT-scan) (Figure 3), the patient can be referred to an otorhinolaryngologist (ENT) for a rigorous consult. If the preventive diagnostic step is omitted, and rhino-sinusal conditions are ignored, it may lead to a disappointing surgery outcome.

The presence of pathological conditions in the nasal-maxillary complex should be considered a contraindication for sinus floor elevation ${ }^{12}$.

Patients guided to see an ENT specialist will undergo a complete cranio-facial examination, with the appreciation of the sinus ventilation. Meanwhile, it is possible to solve any pathological processes or anatomical alterations, preparing the sinus for the dental surgeon. This is an important step in the course of the oral rehabilitation plan.

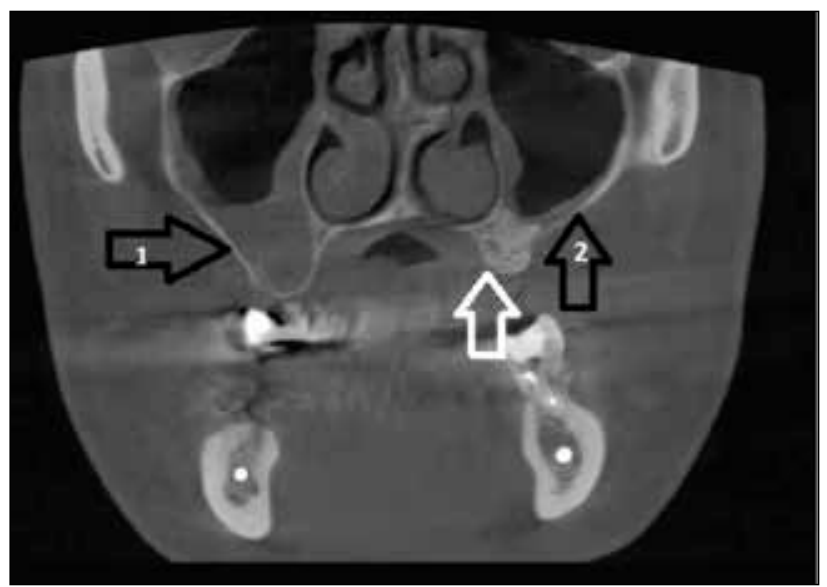

Figure 3 Cranio-facial CT scan, coronal slice - enlarged right maxillary sinus, with chronic sinusitis (arrow 1); successful bone grafting of the left maxillary sinus (white arrow); slender inflamation of the endosinusal mucosa (arrow 2) 
The sinus-lift technique is simple ${ }^{13}$, but the maxillary sinus disease may increase the difficulty of performing sinus lift surgery and the risk of developing postoperative complications ${ }^{14-16}$.

Nasal endoscopy is an important part of the nasosinusal disease diagnostic algorithm ${ }^{17}$. Diagnostic endoscopy allows the visualization of the sinus maxillary cavity and the mucosal changes. This investigation also allows the implantologist to be informed about the endosinusal mucosa aspect. 4-6 weeks after the therapeutic sinusoscopy, the oral surgeon can perform the sinus lift and can insert the implants.

The purpose of this study is to emphasize the diagnostic and therapeutic role of the maxillary sinus endoscopy, in patients with CT scan changes even if they are symptomatic or not.

Treating the maxillary sinus pathology by endoscopic approaches, prior to implant insertion or/and sinus augmentation, is crucial for a better outcome of the dental procedure.

\section{MATERIAL AND METHODS}

Between August 2011 and July 2014, 79 adult patients referred for dental implants were evaluated.

Preoperative, each patient performed a radiologic examination (orthopantomography - OPG). Depending on the width or aspect of the alveolar crest, the pneumatization of the maxillary sinus and the radiological aspect of the sinus mucosa, we established that 34 patients had sinus-lift indication.

This surgical intervention must undergo a preoperative protocol that includes a facial CT scan. CT imaging was abnormal for 15 of our patients. They were referred to an ENT evaluation and, even if some of the patients were symptom-free, the specialist considered all of them to be candidates for maxillary sinusoscopy.

We performed a prospective clinical study including these 15 patients ( 9 women and 6 men) ongoing maxillary sinus augmentation prior to dental implant, having transnasal sinusoscopy (performed by an ENT surgeon) indication after performing the CT scan. We included only adult patients, who consented to the surgical procedures; their age was between 20 and 55 years.

Transnasal endoscopic maxillary view was performed by an ENT surgeon, under local anesthesia, in some cases assisted by intravenous analgosedation. After having the patient's written consent for the sinusoscopy, local anesthesic was applied on the mucosa of the inferior meatus - we used lidocaine solution $10 \%$ in combination with naphazolinum or other nasal decongestants. We preferred this diameatic transnasal approach, rather than canine fossa ap- proach, leaving untouched the anterior sinus wall the access region for the sinus lift intervention. Pressing and using a rotational movement, the trocar and the cannula are inserted in the maxillary cavity, through the medial sinusal wall. The trocar is removed while holding the cannula inside the sinus. The endoscope (with different angulations: $0^{\circ}, 30^{\circ}$, $70^{\circ}$ ) must be inserted for the first time before suction. At the end of the procedure a haemostatic sponge is placed at the inferior meatus.

The aim of the procedure was to examine the aspect of the maxillary sinus mucosa, as well as the presence of cystic dilatation, fungal material or foreign bodies.

\section{RESULTS}

15 patients with maxillary sinus augmentation indication, 9 women and 6 men (female:male ratio=1.5:1), with modified maxillary sinus aspect on the CT scan, participated in our study.

After the clinical assessment and the paraclinical investigations, with the patient consent, we performed an endoscopic sinus evaluation by transnasal approach, through sinusoscopy. The role of the sinus endoscopy is not only to visualize the sinus mucosa, but it may also represent a therapeutic option.

The maxillary sinusoscopy revealed cysts, fungal material, hypertrophic mucosa and normal sinusal aspect (discrepant clinical aspect versus CT imaging) (Table 1).

In five patients, we identified cystic formations, with variable dimensions, located in the alveolar maxillary recess - they were removed transcannular or/and via the new opening located at the inferior meatus (inferior meatotomy).

The fungal material was found in the maxillary sinus of two of the studied patients. In these cases, we preferred to convert to general anesthesia and we en-

$\begin{aligned} & \text { Table } 1 \\
& \text { Endoscopic evaluation of the maxillary sinus (by } \\
& \text { transnasal sinusoscopic approach) }\end{aligned}$
\begin{tabular}{cc} 
Endosinusal Findings & Number of Patients \\
\hline Cyst & 5 \\
\hline Fungal material & 2 \\
\hline Hypertophic/Hyperplasic mucosa & 4 \\
\hline Normal mucosa & 4 \\
\hline TOTAL & 15 \\
\hline
\end{tabular}


tirely removed the fungus ball by performing an antrostomy, with the clearance of the maxillary cavity by suction and saline washing.

In four cases, we encountered dysplasic aspect of the endosinusal mucosa - tissue samples were harvested and sent to the anatomopathological examination.

Inconsistent findings were discovered in four of the patients included in our study group: as the CT scan described an opacified maxillary sinus, thickened mucosa or serous cysts, the endoscopic aspect of the sinusal mucosa was normal, without pathologic secretions. In these cases, we can state that the CT scan images observed only a transitory mucus accumulation, with the subsequent clearance of the secretions, or the presence of an inflammatory process that cured itself physiologically, as the natural maxillary drainage ostium was permeable.

\section{DISCUSSIONS}

Apart from clinical evaluation of the endoscopic aspect of the mucosa, as this method involves a certain subjectivity, we preferred to assess the mucosa status, in an objective manner by using the histopathological evaluation - fragments were collected during sinusoscopy and sent to the anatomopathologist, generating a histopathological score ${ }^{18}$.

Regarding the aspect of endosinusal mucosa, we used a classification, which has been recently proposed as a useful guideline for establishing the course of the treatment, dividing the transformed mucosa into five histopathological types ${ }^{17}$ :

$>$ Type 0 - normal mucosa and vascular pattern, sinus without secretions; the mucolilliary and ostial function are normal.

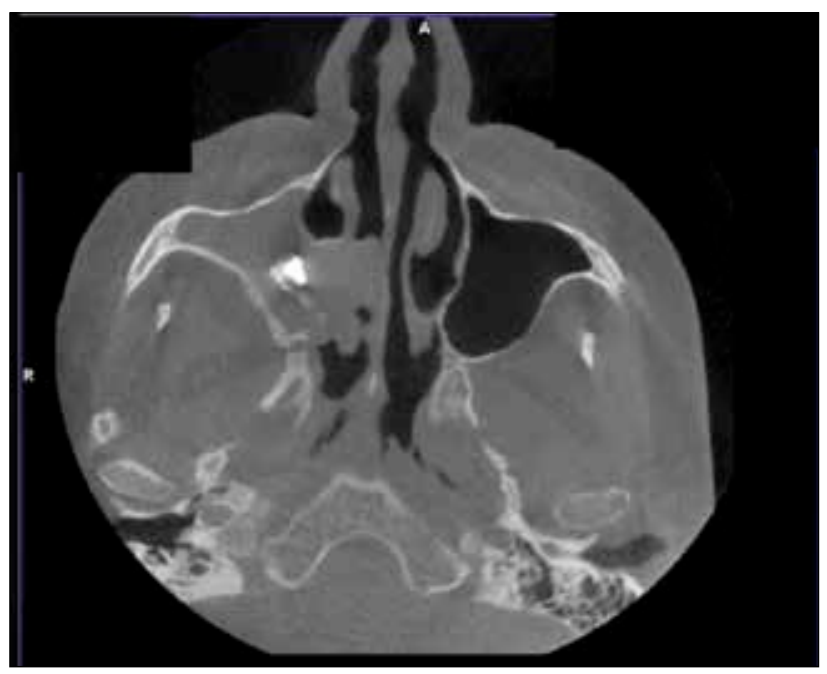

Figure 4 CT scan (axial slice): foreign body in the right maxillary sinus, causing isolated sinusitis, with secretions and hypertrophic mucosa
Type I- pronounced vascular marking, a little bit "dull", with a few serous secretions.

$>$ Type II - thick mucosa, substantial secretions, marked vascular design, dilated glandular ducts, corium alteration. In this stage, this modification responds to medication or to minimal surgical procedures directed to ostial permeabilization.

$>$ Type III - intense modification of the mucosa: polyps, cysts, mucopurulent secretions. Only endoscopic sinus surgery will help.

$>$ Type IV-hyperplasic and metaplasic mucosa, organised polyps, fungosities and caseum. In this case the endoscopic surgery has a poor outcome; often radical classic surgery (Caldwell-Luc technique) is indicated.

The benefits of sinusoscopic procedure consist in: direct visualization and access to the sinusal content, optic evaluation of the integrity and aspect of the mucosa, harvesting biological material and histopathological samples (if needed), instillation of locally-active drugs ${ }^{19}$, foreign body removal or cysts excision. Maxillary sinusoscopy offers a panoramic view, different from the direct normal view ${ }^{17}$. The most common access way is the inferior meatus, perforating the lateral nasal wall - a bony septum that separates the maxillary sinus from nasal cavity ${ }^{20}$.

In some cases, it is indicated to convert the surgical procedure, from transnasal diameatic sinusoscopy to functional endoscopic sinus surgery (enhancing the natural ostium by antrostomy).

As the literature data indicate, inside the maxillary sinus we can encounter some pathological aspects that will interfere with the performance of sinus lift surgery ${ }^{21}$ :

- Foreign bodies: Kerr needles, dental material from root canal obturation (Figure 4), pieces of dental roots, migrated implants

- Fungoid material

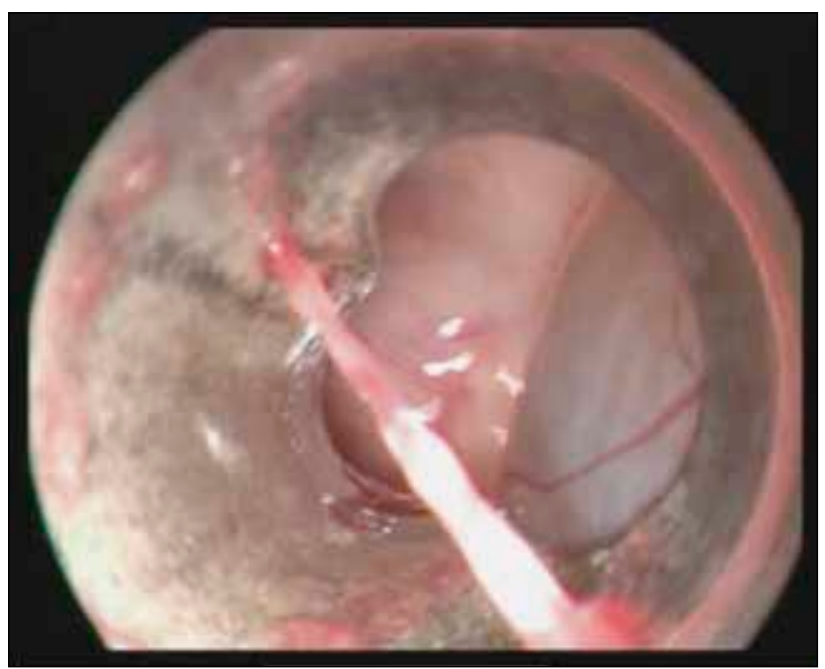

Figure 5 Sinusoscopic view - endosinusal cyst, removed by the ENT specialist through the cannula 


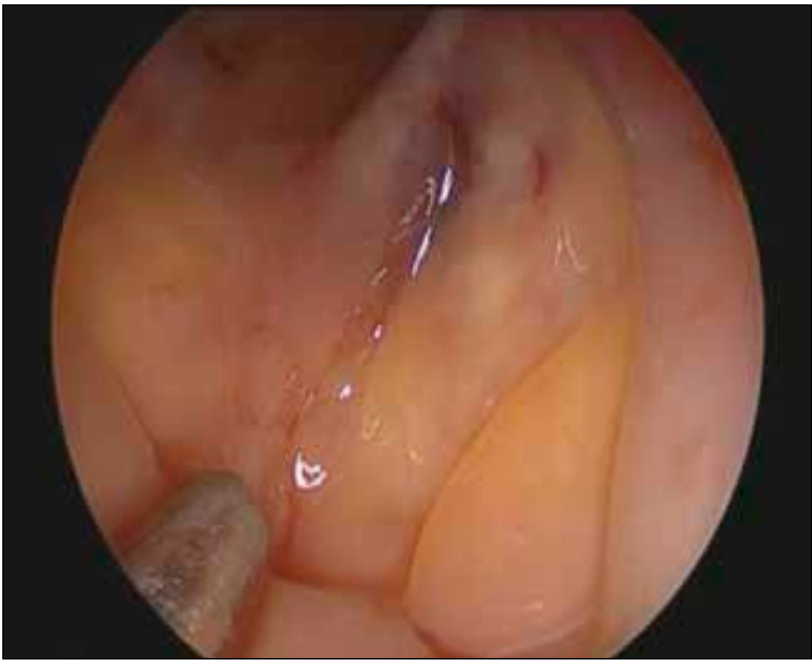

Figure 6 Endoscopic aspect - Polypid degenerated mucosa inside the maxillary sinus

- Mucoceles

- Purulent secretions - acute sinusitis

- Odontogenic / retention cysts or pseudocysts ${ }^{21}$ (Figure 5)

- Hypertrophic or polipoid degenerated mucosa - chronic rhinosinusitis (Figure 6).

Some recent case reports have demonstrated that pseudocysts in the maxillary sinus are not a contraindication for sinus augmentation ${ }^{22,23}$.

Generally, there are no absolute contraindications for transnasal sinusoscopy, only a few relative ones, like the following:

- Hypoplastic sinus - it is observed in $5 \%$ to $10 \%$ of cases $^{24}$

- Inferior narrowed sinus

- Children - sinusoscopy is not indicated for patients under 9-10 years old, in order to prevent harming the dental buds ${ }^{17}$.

During the intervention, there are some accidents and incidents that may occur, as follows ${ }^{17}$ :

- Syncope,

- Inability to enter the maxillary sinus on account of improper technique or the wall is made of compact bone,

- Epistaxis,

- Damage of the lacrimo-nasal duct, the orbit or the posterior wall - by entering in the pterigopalatine fossa.

In our study group we did not encounter any significant complications, just minor bleeding, that did not need surgical or medical intervention.

Due to the limited number of patients that we evaluated, we could not obtain any statistically significant results. The current study is still undergoing, in order to obtain accurate data that may stand as a reference for further preoperative protocols in case of sinus lift interventions.

\section{CONCLUSIONS}

In the management of patients with indication of sinus lift, collaboration between the oro-maxillofacial surgeon and ENT specialists is recommended, in order to improve postoperatory outcomes. The role of ENT specialist is to establish a correct diagnosis before sinus elevation and to provide a suitable treatment, if needed.

To avoid the loss of the graft and inserted implants, in cases with sinus pathology, it is necessary a surgical treatment, able to promptly restore the maxillary sinus ventilation and drainage. Also, treatment can prevent the major complications occurred when implants or grafts are placed into an infected sinus - in this way purulent events with venous septic thrombosis (that may follow) are limited.

The best approach consists in performing a transnasal endoscopy, which is a safe and useful method, in the hands of an experienced ENT surgeon.

\section{REFERENCES}

1. McCaul L.K., Jenkins W.M., Kay E.J. - The reasons for the extraction of various tooth types in Scotland: a 15-year follow up. J Dent., 2001;29:401407.

2. Thomson W.M. - Monitoring edentulism in older New Zealand adults over two decades: A review and commentary. Int J Dent., 2012;2012. doi:10.1155/2012/375407.

3. Muller F., Naharro M., Carlsson G.E. - What are the prevalence and incidence of tooth loss in the adult and elderly population in Europe? Clin Oral Implants Res., 2007;18 Suppl 3:2-14.

4. Douglass C.W., Shih A., Ostry L. - Will there be a need for complete dentures in the United States in 2020? Journal of Prosthetic Dentistry, 2002;87(1):5-8.

5. Carlsson G.E., Bergman B., Hedegard B. - Changes in contour of the maxillary alveolar process under immediate dentures. A longitudinal clinical and x-ray cephalometric study covering 5 years. Acta Odontol Scand., 1967;25:45-75.

6. Pietrokovski J., Massler M. - Alveolar ridge resorption following tooth extraction. J Prosthet Dent., 1967;17:21-27.

7. Kelsey C.C. - Alveolar bone resorption under complete dentures. J Prosthet Dent.,1971;25:152-161.

8. Pietrokovski J., Starinsky R., Arensburg B., Kaffe I. - Morphologic characteristics of bony edentulous jaws. J Prosthodont., 2007;16:141-147.

9. Wehrbein H., Diedrich P. - Progressive pneumatization of the basal maxillary sinus after extraction and space closure. Fortschr Kieferorthop, 1992;53:77-83.

10. Sharan A., Madjar D. - Maxillary sinus pneumatization following extractions: a radiographic study. Int J Oral Maxillofac Implants, 2008;23:4856.

11. Johanson L.Å. - On minimally invasive approaches to sinus lift procedures. Sweden. 2012. Available from: http://hdl.handle.net/2077/30265.

12. Beaumont C., Zafiropoulos G.G., Rohmann K., Tatakis D.N. - Prevalence of maxillary sinus disease and abnormalities in patients scheduled for sinus lift procedures. J Periodontol., 2005;76:461-467.

13. Bucur A. - Compendium de chirurgie oro-maxilo-faciala. Volumul I. Editura Q Med Publishing, Bucuresti, 2009. 
14. Manor Y., Mardinger O., Bietlitum I., Nashef A., Nissan J., Chaushu G. Late signs and symptoms of maxillary sinusitis after sinus augmentation. Oral Surg Oral Med Oral Pathol Oral Radiol Endod., 2010;110:e1-4.

15. Anavi Y., Allon D.M., Avishai G., Calderon S. - Complications of maxillary sinus augmentations in a selective series of patients. Oral Surg Oral Med Oral Pathol Oral Radiol Endod., 2008;106:34-38.

16. Timmenga N.M., Raghoebar G.M., Boering G., van Weissenbruch R. Maxillary sinus function after sinus lifts for the insertion of dental implants. J Oral Maxillofac Surg., 1997;55:936-939; discussion 940.

17. Sarafoleanu C. - Rinologie. Editura Medicala, Bucuresti, 2003;pp.83-103.

18. Manea C., Iosif C., Sarafoleanu D. - The value of histopathologic score as predictive factor in choosing the optimal surgical treatment for chronic rhinosinusitis. Romanian Journal of Rhinology, 2012;2(6):90-95.

19. Sarafoleanu C. - Examenul endoscopic al nasului si sinusurilor paranazale. In: sub red. Sarafoleanu D. - Explorarea paraclinica si functionala in
Otorinolaringologie. Vol I. Editura didactica si Pedagogica, Bucuresti, 1998;pp.26-34.

20. Testori T., del Fabbro M., Weinstein R., Wallace S. - Maxillary sinus surgery and alternatives in treatment. Quintessence Pub Co Ltd., 2009.

21. Chan H.L., Wang H.L. - Sinus pathology and anatomy in relation to complications in lateral window sinus augmentation. Implant Dent., 2011;20:406-412.

22. Brook I. - Sinusitis of odontogenic origin. Otolaryngol Head Neck Surg., 2006;135:349-355.

23. Abrahams J.J., Glassberg R.M. - Dental disease: a frequently unrecognized cause of maxillary sinus abnormalities? AJR Am J Roentgenol., 1996;166:1219-1223.

24. Watzek G. - The Precrestalsunus lift. From illusion to reality. London, UK, 2012;pp.3-14 